\title{
Experimental Investigation of High-Enthalpy Effects on Attachment-Line Boundary-Layer Transition
}

\author{
Keisuke Fujii* \\ National Aerospace Laboratory, Tokyo 182-8522, Japan \\ and \\ Hans G. Hornung ${ }^{\dagger}$ \\ California Institute of Technology, Pasadena, California 91125
}

\begin{abstract}
A series of experiments was performed on high-enthalpy effects on hypersonic boundary-layer transition using nitrogen, air, and carbon dioxide as test gases in the T5 hypervelocity shock tunnel.Previous experiments on a sharp cone showed significant high enthalpy effects. This series concerns the attachment line boundary layer on swept cylinders with sweep angles of 60 and $45 \mathrm{deg}$. The observed trend of transition Reynolds number with enthalpy, which is found to be similar to that in the cone results, shows strong transition delay at the larger sweep angle for carbon dioxide, whereas no significant effect is observed in nitrogen and air. The acoustic wave absorption rate due to relaxation shows a quite similar trend in enthalpy dependence to transition Reynolds number for the carbon dioxide case. This suggests that the dominant effect in delaying transition in the case is vibrational relaxation. The comparisons between the magnitude of the strongest amplification rate from an inviscid linear stability analysis and the absorption rate due to relaxation show that they are of the same order of magnitude for carbon dioxide and that absorption is not significant for nitrogen or air, which supports the stated understanding of the effect of relaxation on transition.
\end{abstract}

\section{Nomenclature}

$=$

$=$

$=$

$=$

$=$

\section{Subscripts}

$=$ edge condition

$=$ recovery condition

$=$ wall condition

$=$ reservoir condition

$=$ freestream condition
Received 15 January 2002; revision received 17 July 2002; accepted for publication 30 September 2002. Copyright (C) 2003 by Keisuke Fujii and Hans G. Hornung. Published by the American Institute of Aeronautics and Astronautics, Inc., with permission. Copies of this paper may be made for personal or internal use, on condition that the copier pay the $\$ 10.00$ per-copy fee to the Copyright Clearance Center, Inc., 222 Rosewood Drive, Danvers, MA 01923; include the code 0001-1452/03 \$10.00 in correspondence with the CCC.

* Researcher, Wind Tunnel Technology Center, Member AIAA.

Clarence L. Johnson Professor of Aeronautics and Director, Graduate Aeronautical Laboratories. Member AIAA.
Superscript

$* \quad=$ reference condition

\section{Introduction}

$\mathbf{C}$ OMPRESSIBLE boundary-layer transition has been a subject of study for many years, but only a limited number of experimental studies exist that were made at high-enthalpy hypersonic speeds, where vibrational and chemical effects become important. One of the earliest experimental studies was done by He and Morgan, ${ }^{1}$ who investigated the boundary layer on a flat plate at 30-deg angle of attack with air as the test gas. They reported that the transition Reynolds number decreases with increasing total enthalpy in the manner of the effect of wall cooling on the second-mode instability under their conditions, which were in the range $5.2<M_{\infty}<6.6$. Some other experimental studies were made by Germain and Hornung ${ }^{2}$ and Adam and Hornung ${ }^{3}$ using a 5-deg half-angle cone model in the T5 hypervelocity shock tunnel. They observed that increasing enthalpy had a slight stabilizing effect in terms of the transition Reynolds number evaluated at the boundary-layer reference condition, in the case of nitrogen and air. Also, it was revealed that enthalpy increase stabilizes the boundary layer dramatically in the case of carbon dioxide.

There have been a number of numerical investigations made on the high-enthalpy effect, including those by Malik and Anderson, ${ }^{4}$ who assumed both thermally and chemically equilibrium airflow, and by Stuckert and Reed, ${ }^{5}$ who considered vibrational equilibrium but chemical nonequilibrium flow. The former found that equilibrium high-enthalpyeffects stabilize first-mode disturbancesand that they destabilize higher modes, whereas the latter found that the chemical nonequilibrium effect stabilizes the boundary layer but not significantly. More recently, Johnson et al. ${ }^{6}$ calculated the linear stability of the boundary layer on a 5-deg half-angle cone considering thermal and chemical nonequilibriumeffects. They examined high-enthalpyeffects on transition throughmean flow profile change and disturbance growth separately. They found that reacting mean flow would have larger amplification than that when no reaction existed, but that finite relaxation rate caused a net damping effect that was especially marked in carbon dioxide. They also made comparisons of their numerical results with the T5 experimental data 
mentioned earlier and observed a consistent tendency in transition Reynolds number dependence on enthalpy for both air and carbon dioxide.

This may also be supported by an analysis introduced by Clarke and McChesney, ${ }^{7}$ who considered effects of relaxation on sound wave propagation in an ideal dissociating gas. They showed that the relaxation in the chemical reaction damps sound waves whose frequency is near the reciprocal of the relaxation time. This suggests that, depending on the relation between the most strongly amplified frequency in the boundary layer and the frequency of relaxation, the effect of high enthalpy may be quite different. In this experiment, therefore, we study transition of the attachment line boundary layer on a swept cylinder of different sweep angles, where a large variation in the relation of these two characteristic frequencies can be achieved.

\section{Background}

\section{High-Enthalpy Effect on Transition}

It is known that transition in hypersonic flow may be initiated by two instability modes: the first mode associated with TollmienSchlichting waves, that is, vortical disturbances, and the second mode associated with an inviscid instability, in the form of trapped acoustic disturbances in the boundary layer. Because the second mode is the dominant instability mode at sufficiently high Mach number, especially for a very highly cooled wall situation (such as in T5 hypervelocity shock-tunnel experiments), it stabilizes the inviscid first mode completely. Because the generalized inflection point vanishes in the boundary-layerprofile, the instability waves in such a boundary layer can be expected to have features of acoustic waves. ${ }^{8}$

It is also known that acoustic waves traveling through a gas are attenuated by relaxation processes. This phenomenon can be recognized as sound suppression due to bulk viscosity when the wave frequency is sufficiently low compared with the relaxation frequency. Around the relaxation frequency, this sound absorption rate reaches a maximum whose magnitude depends on the ratio of the frozen sound speed (for infinitely high-frequency waves) $a_{f}$ to the equilibrium sound speed (for infinitesimally low-frequency waves) $a_{e}$. Generally, the frozen sound speed $a_{f}$ is faster than the equilibrium sound speed $a_{e}$ so that greater ratios $a_{f} / a_{e}$ cause greater damping effects.

As has been pointed out by Lighthill, ${ }^{9}$ the bulk viscosity of a diatomic gas at room temperature arises from the rotational relaxation process, which has a characteristicfrequency of approximately $1 \mathrm{GHz}$ at $p=1 \mathrm{~atm}$ for nitrogen.For high-temperaturegases, such as the case of our experiment, this frequency will become even higher. However, the frequencies of vibrational and/or chemical relaxation might become interesting because the most strongly amplified frequency in the boundary-layerranges approximately between 1 and $10 \mathrm{MHz}$. The relaxation time for vibrational excitation is a function of temperature and pressure. An approximation for it can be written as ${ }^{10}$

$$
\ln \tau=(K / T)^{\frac{1}{3}}-\ln p+\ln C
$$

The physics and estimation procedure for the absorption are described in detail by textbooks such as those by Clarke and McChesney $^{7}$ and Vincenti and Kruger ${ }^{10}$ for the case where only one relaxing mode exists. In practical situations, however, there exists more than one relaxing mode. Figure 1 shows sound absorption rate per wavelength $\alpha_{\text {relax }} \lambda$ defined by

$$
\phi=\phi_{0} \exp \left[-\alpha_{\text {relax }} \lambda(x / \lambda)\right] \exp \left[i\left(\alpha_{i} x-\omega t\right)\right]
$$

for nitrogen, air, and carbon dioxide at constant density of $0.1 \mathrm{~kg} / \mathrm{m}^{3}$ and at temperature ranging from 2000 to $6000 \mathrm{~K}$ for nitrogen and 1000 to $5000 \mathrm{~K}$ for carbon dioxide and air. (A description of the method of calculating these may be found in Ref. 11.) Figures $1 \mathrm{a}-1 \mathrm{c}$ show that sound absorption is quite strong for a range of frequencies at sufficiently high temperature and that the absorption curve exhibits several humps at some temperatures.

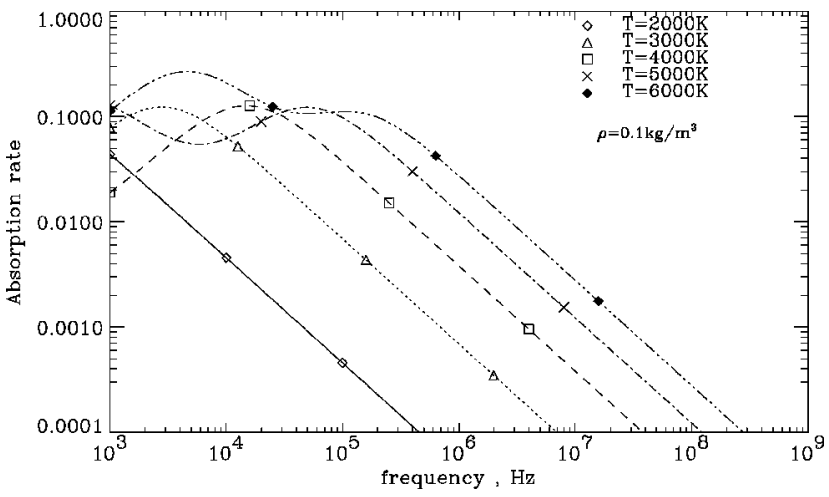

a) Nitrogen

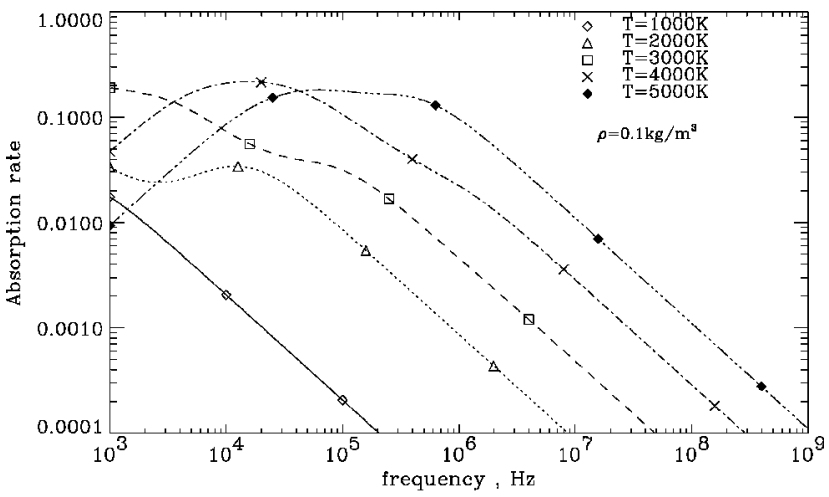

b) Air

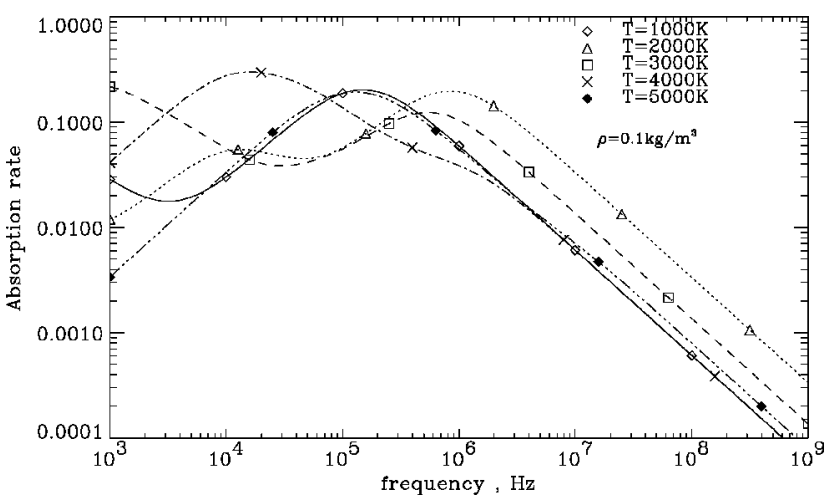

c) Carbon dioxide

Fig. 1 Sound absorption rate per wave length due to relaxation of chemical reactions and vibrational excitation vs frequency; density kept constant at $0.1 \mathrm{~kg} / \mathrm{m}^{3}$ to represent approximately experimental conditions.

The absorption peak at the higher frequency corresponds to vibrational relaxation and the other to chemical relaxations. Note that, in the case of carbon dioxide, the value of the absorption maximum associated with vibrational relaxation decreases as the temperature becomes sufficiently high because of dissociation of $\mathrm{CO}_{2}$ molecules.

Suppose the most strongly amplified wave to be an acoustic wave; the idea suggests itself that boundary-layer transition might be delayed by the damping effect due to relaxation processes. Thus, the frequency of the most strongly amplified second-mode disturbance in relation to the characteristic frequency of the relaxation process becomes important. The frequency may be estimated roughly by a simple form ${ }^{12}$ for second-mode disturbances: 
Because the magnitude of the sound absorption rate depends monotonically on $a_{f} / a_{e}-1$, sound waves would be amplified by relaxation processes if the frozen sound speed were smaller than the equilibrium sound speed. This could be examined in numerical calculations, for example, by switching the sign of the enthalpy derivative with respect to the degree of dissociation for ideal dissociating gas, as was done by Johnson et al. ${ }^{6}$ Their numerical result that this virtual switching leads to a significant destabilization underlines the importance of relaxation processes on disturbance growth.

From Eqs. (1) and (2), a ratio of the two frequencies, $1 / \tau f_{2 \text { nd }}$, which defines a Damköhler number, can be written as

$$
\frac{1}{\tau f_{\text {2nd }}} \approx \frac{2 \delta p_{e}}{u_{e} C \exp \left[\left(K / T^{*}\right)^{\frac{1}{3}}\right]} \approx \frac{2 \mu^{*} R e_{\delta}^{*}}{\gamma^{*} M_{e}^{* 2} \exp \left[\left(K / T^{*}\right)^{\frac{1}{3}}\right]}
$$

where the asterisk denotes the value evaluated at a representative temperature in the boundary layer, which will be chosen to be that of the reference enthalpy condition later. Because Eq. (3) is only a rough estimate, we used the perfect gas relation here. From Eq. (3), assuming that transition occurs at a constant Reynolds number $R e_{\delta}^{*}$, the Damköhler number increases as the edge Mach number $M_{e}^{*}$ decreases or as the reference temperature increases. Thus, roughly speaking, the Damköhler number is expected to increase with decreasing sweep angle.

\section{Previous Experiments in T5}

A summary of the results of the sharp cone experiments of Germain and Hornung ${ }^{2}$ and Adam and Hornung ${ }^{3}$ is shown in Fig. 2, which also includes additionalresults from Rasheed. ${ }^{8}$ The Reynolds number here is based on distance of transition location from the cone tip and local flow parameters evaluated at Eckert's reference temperature. ${ }^{13}$ Figure 2 shows two major features: First, the transition Reynolds number varies with total enthalpy by almost a factor of 10 for carbon dioxide and, second, the transition Reynolds number in air increases with total enthalpy and is slightly larger than that of nitrogen. When the boundary-layerthickness is estimated as will be described later, the sound absorption rate due to relaxation processes is calculated at the frequency of Eq. (2) for the conditions of the experiment by Rasheed ${ }^{8}$ and is shown in Fig. 3. As may be seen, the absorption rate is up to three orders of magnitude larger in carbon dioxide than in nitrogen. For nitrogen, the Damköhler number [Eq. (3)] is very small, so that vibrational relaxation of nitrogen is very weak at the most strongly amplified frequency and the damping effect is much reduced. In the case of air, the vibrational excitation of oxygen is expected to produce a stronger damping effect than in pure nitrogen.

\section{Attachment Line Transition}

As was pointed out earlier, the attachment-line boundary layer on a swept cylinder provides the opportunity not only to obtain a

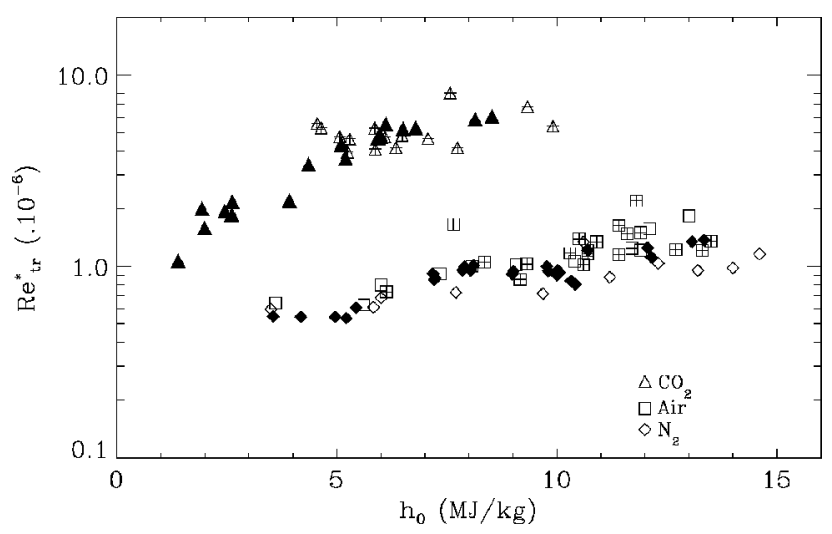

Fig. 2 Transition Reynolds number observed in previous cone experiments: open symbols, from Germain et al. $^{2}$; +, from Adam et al..$^{3}$; and solid symbols, from Rasheed. ${ }^{8}$

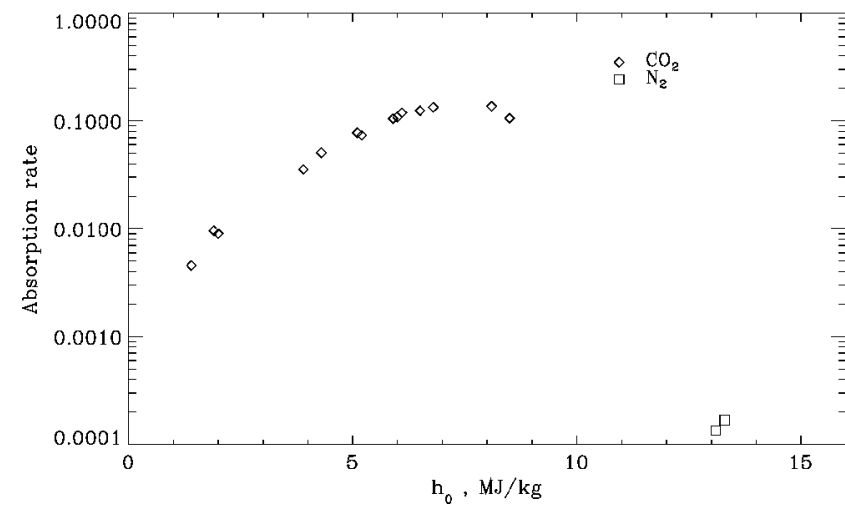

Fig. 3 Sound absorption rate per wave length at reference condition at the most strongly amplified frequency in 5-deg half-angle cone flow with reference condition cited from Rasheed. ${ }^{8}$ Most strongly amplified frequencies are estimated with Eq. (2), where boundary-layer thickness is determined from results of BLIMPK ${ }^{25}$ calculations.

very different value of the Damköhler number than in the flow over a sharp cone but also to vary it by changing the sweep angle. To evaluate the transition condition for the attachment-line boundary layer on a swept cylinder without chemistry, the local Reynolds number proposed by Poll, ${ }^{14} \bar{R} e$, which is based on the boundarylayer characteristiclength $\eta$ and edge velocity in the axial direction, are commonly used in incompressibleflow. The characteristiclength for that Reynolds number is defined as

$$
\eta=\sqrt{\mu_{e} / \rho_{e} \frac{\mathrm{d} w_{e}}{\mathrm{~d} z}}
$$

Poll ${ }^{15}$ also extended this notation to compressibleflow by evaluating the density and viscosity in the Reynolds number at a reference temperature in the boundary layer, thus, introducing $\bar{R} e^{*}$, where the reference temperature here is defined by

$$
T_{\text {Poll }}^{*}=0.10 T_{w}+0.60 T_{r}+0.30 T_{e}
$$

Many experimental and numerical investigations on attachmentline transition in perfect gas flows have been made. Benard et. al. ${ }^{16}$ gives a good summary of the literature in this area. Creel et al., ${ }^{17}$ studied these flows in the low-disturbancesupersonic wind tunnel at NASA Langley Research Center. They reported the upper limit of the value of $R e^{*}$ at which transition occurs without roughness as ranging between 650 and 750 , which is approximately the same value as for "noisy" freestream conditions.

\section{Experiment}

A series of experiments was conducted in the T5 hypervelocity shock tunnel at the California Institute of Technology. This facility is a free-piston shock tunnel designed to obtain high-enthalpy and relatively high-Reynolds-numberflow. The ranges of the reservoir and freestream conditions for each gas in this experiment are summarized in Table 1. When the large extent of the reservoir condition range was considered, a conical nozzle was used all through the tests so that reasonably good flow quality is obtained. To extend the Reynolds number range at the low end in the case of low-enthalpy flow, the 30-mm-diam nozzle throatused for most of the experiments was replaced by a $15-\mathrm{mm}$-diam throat. The conical flow creates a longitudinal pressure gradient, which can affect the boundary-layer transition. This would be an important concern for our investigation if this pressure gradient were dependent on the total enthalpy or on the test gas. A plot of the longitudinal pressure gradient as calculated by a one-dimensional nonequilibrium nozzle flow code for each shot conditions does not show any clear dependence on total enthalpy or test gas (Fig. 4).

Nitrogen, oxygen, and carbon dioxide are expected to be excited vibrationally and to react chemically (for air and carbon dioxide 
Table 1 Summary of flow conditions

\begin{tabular}{|c|c|c|c|c|c|c|c|c|}
\hline Gas & $\begin{array}{c}\text { Throat } \\
\text { diameter, } \mathrm{mm}\end{array}$ & $\begin{array}{c}h_{0}, \\
\mathrm{MJ} / \mathrm{kg}\end{array}$ & $\begin{array}{l}P_{0} \\
\mathrm{MPa}\end{array}$ & $\begin{array}{l}U_{e} \\
\mathrm{~m} / \mathrm{s}\end{array}$ & $M_{e}$ & $\begin{array}{c}T_{e}, \\
\mathrm{~K}\end{array}$ & $\begin{array}{c}\rho_{e} \\
\mathrm{~kg} / \mathrm{m}^{3}\end{array}$ & $\begin{array}{l}\text { Mole fraction } \\
\text { at the edge }\end{array}$ \\
\hline \multicolumn{8}{|c|}{$\Lambda=60 \mathrm{deg}$} & $x_{\mathrm{N}_{2}}$ \\
\hline $\mathrm{N}_{2}$ & 30 & $2.0-15.0$ & $10.0-71.9$ & $1660-4300$ & $3.3-3.4$ & $620-4480$ & $0.07-0.37$ & $0.99-1.00$ \\
\hline $\mathrm{N}_{2}$ & 30 & $18.7-20.7$ & $53.7-57.5$ & $4690-4880$ & 3.2 & $5350-5660$ & 0.15 & $0.91-0.95$ \\
\hline Air & 30 & $1.7-15.9$ & $11.9-68.5$ & $1650-4300$ & $3.2-3.3$ & $640-3930$ & $0.12-0.28$ & $\begin{array}{c}x_{\mathrm{N}_{2}} / x_{\mathrm{O}_{2}} \\
0.67-0.78 / 0.06-0.21\end{array}$ \\
\hline $\mathrm{CO}_{2}$ & 15 & $1.5-4.3$ & $4.4-48.6$ & $1410-2080$ & $3.2-3.7$ & $760-1940$ & $0.02-0.31$ & $\begin{array}{c}x_{\mathrm{CO}_{2}} \\
0.97-1.00\end{array}$ \\
\hline $\mathrm{CO}_{2}$ & 30 & $1.4-4.4$ & $4.7-47.8$ & $1400-2130$ & $3.2-3.4$ & $750-1960$ & $0.09-0.68$ & $0.98-1.00$ \\
\hline $\mathrm{CO}_{2}$ & 30 & $5.8-15.4$ & $23.0-72.2$ & $2360-3540$ & 3.3 & $2320-3440$ & $0.20-0.43$ & $0.22-0.92$ \\
\hline \multicolumn{8}{|c|}{$\Lambda=45 \mathrm{deg}$} & $x_{\mathrm{N}_{2}}$ \\
\hline $\mathrm{N}_{2}$ & 30 & $3.3-16.5$ & $6.3-66.5$ & $1800-3660$ & $2.2-2.3$ & $1760-6090$ & $0.13-0.31$ & $0.88-1.00$ \\
\hline Air & 30 & $2.8-16.5$ & $6.3-66.5$ & $1640-3550$ & $2.1-2.3$ & $1590-5310$ & $0.03-0.36$ & $\begin{array}{c}x_{\mathrm{N}_{2}} / x_{\mathrm{O}_{2}} \\
0.62-0.78 / 0.01-0.21\end{array}$ \\
\hline $\mathrm{CO}_{2}$ & 30 & $2.2-16.2$ & $3.8-60.9$ & $1340-2910$ & 2.4 & $1440-3840$ & $0.12-0.64$ & $\begin{array}{c}x_{\mathrm{CO}_{2}} \\
0.11-1.00\end{array}$ \\
\hline
\end{tabular}

${ }^{a}$ Mole fraction is at edge of attachment-line boundary layer assumed in equilibrium.

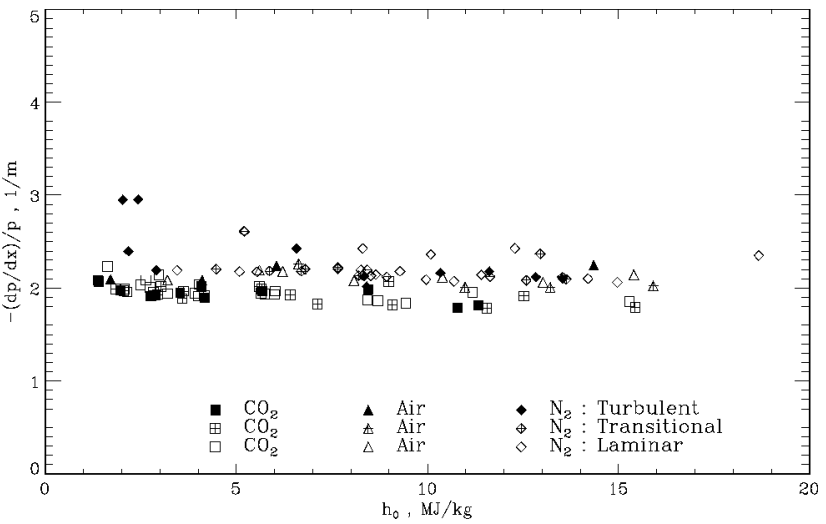

Fig. 4 Longitudinal pressure gradient in freestream; no evident dependence on the total enthalpy or on the test gas is seen.

tests) in the range of conditions of this experiment. The swept cylinder models have cylinderdiameters of 50.8 and $50.19 \mathrm{~mm}$ and sweep angle of 45 and 60 deg, respectively, as shown in Fig. 5. They are equipped with coaxial thermocouples to measure the heat transfer rate from the time dependence of surface temperature on the attachment line. The boundary-layer state was detected by comparing the measured heat transfer with the theoretical prediction for both laminar and turbulentattachment-lineheat transfer as proposed by Beckwith and Gallagher. ${ }^{18}$ Examples of heat flux traces measured at laminar, intermittent, and turbulent conditions are shown in Fig. 6. The high heat flux period at the beginning of each shot, which is approximately up to $1 \mathrm{~ms}$, represents the starting process during which the nozzle flow is established. The test time is taken as the time between the end of the starting process and the onset of driver gas contamination, as indicated by a set of dashed vertical lines in Fig. 6. The heat flux averaged during the test time is compared to the prediction to determine the boundary-layer state. Figure 7, the averaged heat flux, shows fairly constant distribution around the point $x / r \approx 10$, where the boundary-layerstate was examined.

Freestream properties are derived by nonequilibriumnozzle flow computation, from measured stagnation pressure and measured shock speed. The flow properties behind the bow shock are calculated assuming thermal and chemical equilibrium based on the JANNAF tables, ${ }^{19}$ because estimated relaxation distances are very small. The viscosity of the mixture at each condition is then calculated by the semi-empirical formula of Wilke ${ }^{20}$ where the viscosity for each component of the mixture is taken from the first-order solution of kinetic theory with the collision integrals approximated by an empirical equation. ${ }^{21}$

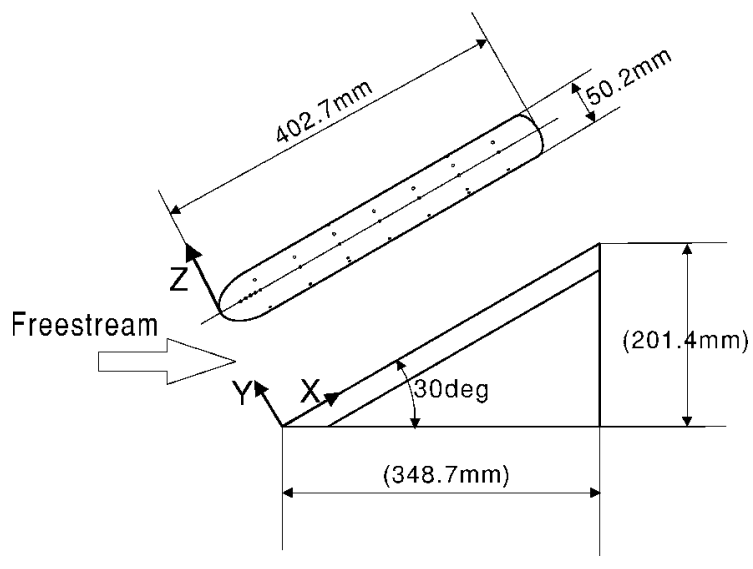

a) $\Lambda=60 \mathrm{deg}$ model

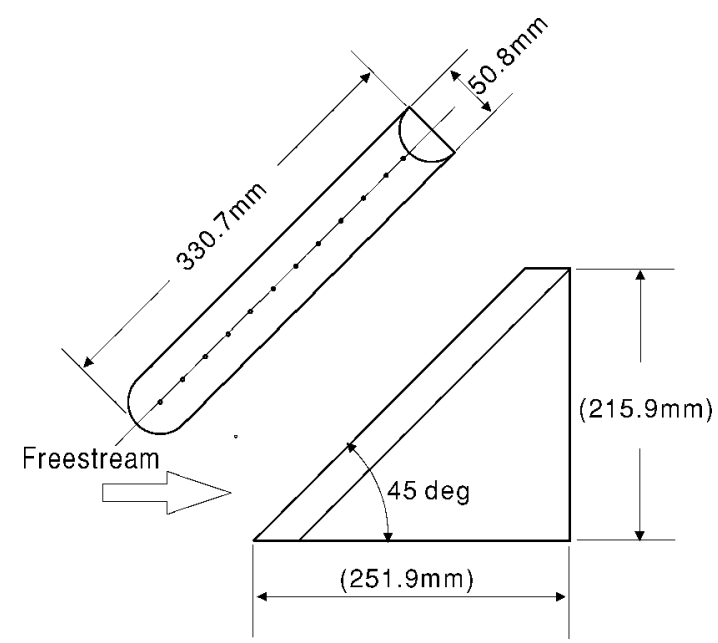

b) $\Lambda=45$ deg model

Fig. 5 Swept cylinder models for this experiment.

The edge Mach number for each shot condition is calculated as low as 3.3 for the 60-deg sweep model and 2.4 for the 45-deg sweep model (Table 1), which is lower than the Mach number where the second-mode instability takes its dominance for the insulated flat plate boundary layer. ${ }^{22}$ According to an early compressible stability theory, however, with sufficient cooling, the first mode can be made completely stable at any Reynolds number (see Ref. 23). In fact, an "inviscid" instability calculation under present experimental conditionsindicates that no unstable first mode exists, due to the wall 


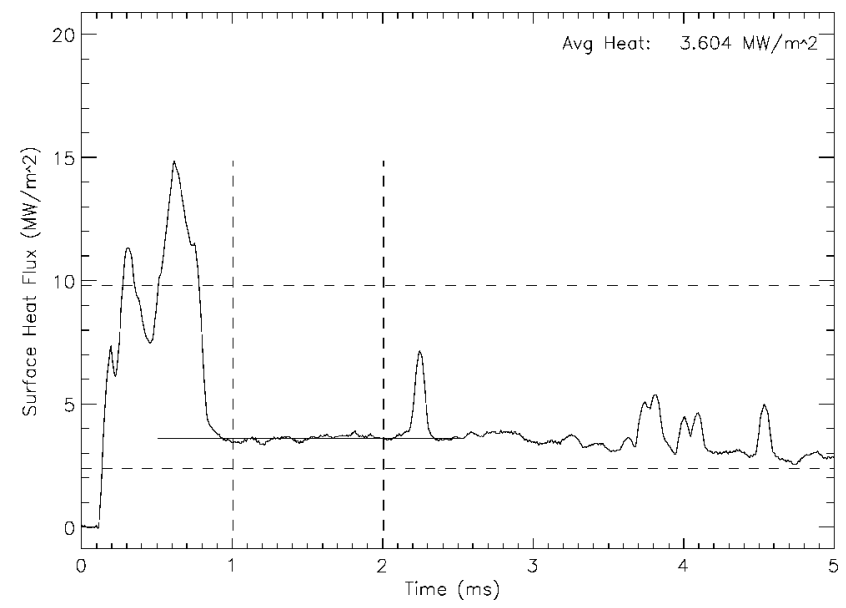

a) Laminar, $\bar{R} e^{*}=893.3$, and $h_{0}=5.8 \mathrm{MJ} / \mathrm{kg}$

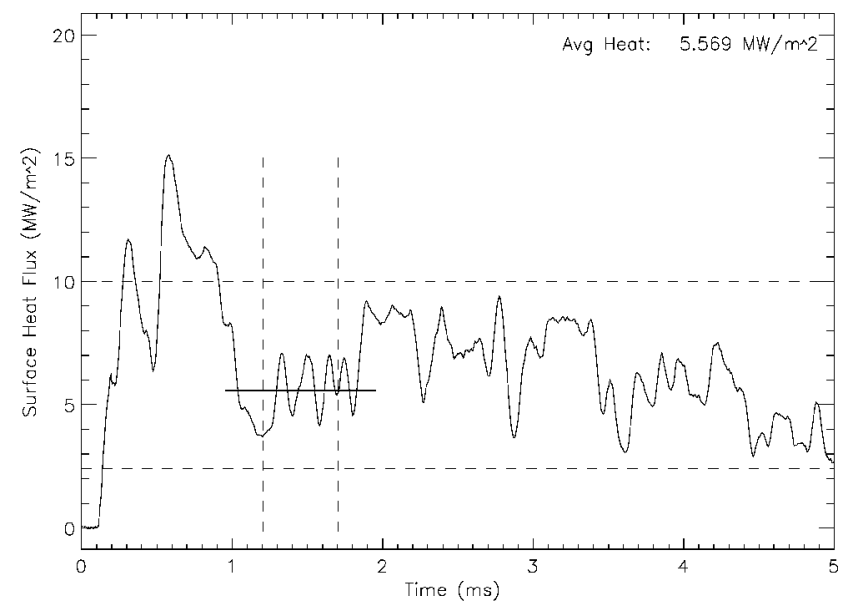

b) Intermittent, $\bar{R} e^{*}=887.2$, and $h_{0}=5.8 \mathrm{MJ} / \mathrm{kg}$

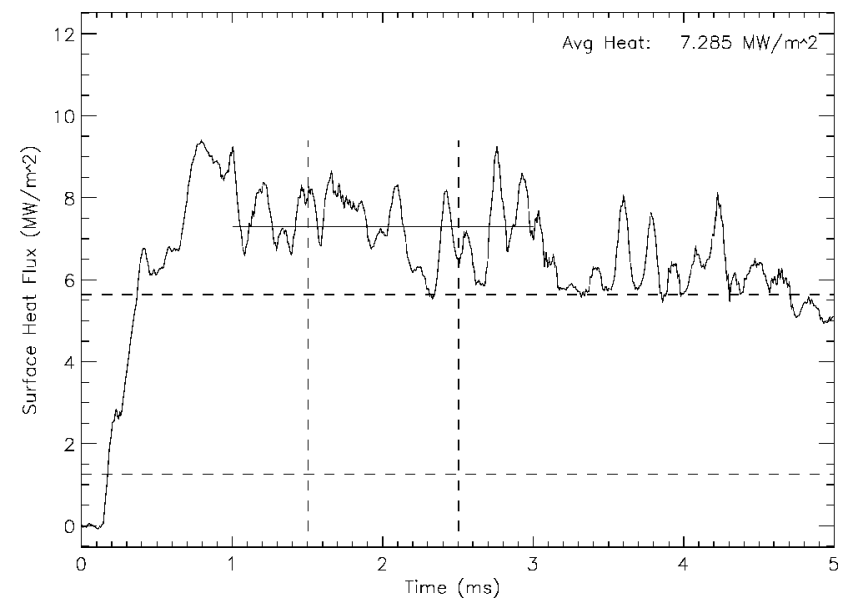

c) Turbulent, $\bar{R} e^{*}=1407.2$, and $h_{0}=3.0 \mathrm{MJ} / \mathrm{kg}$

Fig. 6 Example of heat flux trace measured at sensor $9(x / r \approx 10)$ of the $\Lambda=60$ deg model with $\mathrm{CO}_{2}$ test gas, shot 2046 .

cooling effect. Although this does not exclude viscous instability, it means that the Mach number where the second mode takes its dominance is expected to be lower in the highly cooled wall condition than in the insulated case.

At the high enthalpy and with the short test time of the experiment, the wall temperature is only a small fraction of the recovery temperature. This causes Poll's reference temperature ${ }^{15}$ calculated from Eq. (5) to be much higher than any actual temperature inside the boundary-layerprofile. Because that reference temperature does not seem to have a physical meaning in this situation anymore,

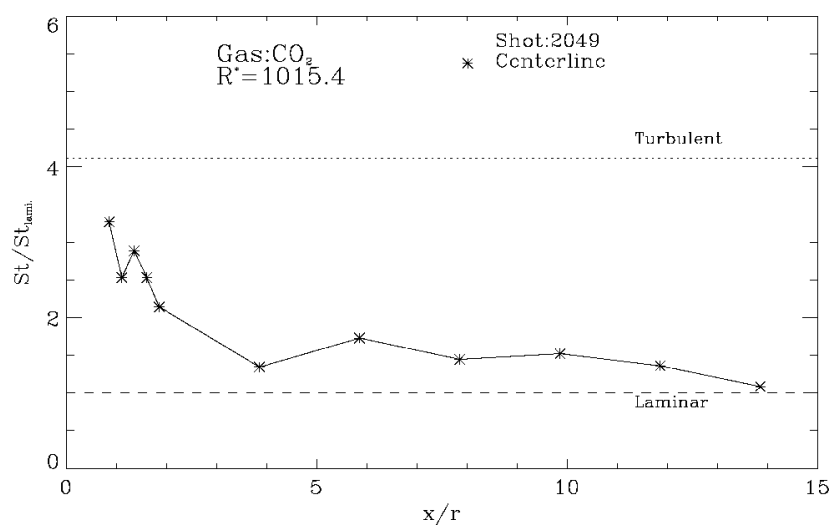

Fig. 7 Distribution of heat flux measured at shot 2049, $\mathrm{CO}_{2}$ test gas, and 60-deg sweep angle.

it was decided to use Eckert's reference enthalpy method ${ }^{13}$ instead to determine the reference properties even for the $\bar{R} e^{*}$ evaluation. As pointed out by Dorrance ${ }^{24}$ this reference enthalpy has the meaning of "mean enthalpy" for the Chapman-Rubesin parameter in the profile. The formula used here for each shot is

$$
h_{\mathrm{Eckert}}^{*}=\frac{1}{2}\left(h_{e}+h_{w}\right)+0.092 u_{e}^{2}
$$

As for the other flow parameters behind the bow shock, the condition is assumed to be in equilibrium. In the carbon dioxide case, however, the composition at the wall $\left(T_{w}=300 \mathrm{~K}\right)$ is not necessarily $100 \%$ carbon dioxide, especially for high-enthalpy shots. This is because the dissociated gas in the boundary layer reacts at the wall along with a set of surface reactions to form a mixture of $\mathrm{CO}_{2}, \mathrm{CO}$, and $\mathrm{O}_{2}$. Thus, the enthalpy at the wall, $h_{w}$, can be higher than what it would be if the gas were to recombine to pure carbon dioxide, even at a temperature as low as $300 \mathrm{~K}$. Thus, the composition at the wall is calculated assuming a fully catalytic wall by a two-dimensional non-equilibriumboundary layer code, BLIMPK, ${ }^{25}$ according to the idea of the axisymmetric analogy proposed by Cooke. ${ }^{26}$ A set of surface reactions are taken from the report by Chen et. al. ${ }^{27}$

\section{Data Analysis}

Sound Absorption Rate at $f_{2 \text { nd }}$

The sound absorption due to the relaxation was estimated at a representative condition in the boundary layer, that is, the reference enthalpy condition defined earlier. The following assumptions were made: 1) translation and rotation in equilibrium, 2) both vibrational excitationsand chemical reactions in finite rate relaxation, and 3 ) electronic excitations to be frozen in equilibrium state. ${ }^{11}$ At the high temperatures of our experiment, the first assumption is good, although vibrational and chemical nonequilibriummay be expected to be active. See also Ref. 28 for lower temperature situations.

The boundary-layerthickness needed in the estimation [Eq. (2)] is taken as the thickness at which the velocity reaches $99 \%$ of the edge velocity, $\delta_{99}$, which is determined from the profile calculated by a two-dimensionalnonequilibriumboundary-layercode, BLIMPK. ${ }^{25}$

\section{Inviscid Linear Instability}

A two-dimensional inviscid linear instability analysis was carried out assuming perfect gas flow for both mean flow profile and disturbances. The mean flow profile is calculated with the method described by Reshotko and Beckwith. ${ }^{29}$ In this analysis, the specific heat ratio $\gamma$ is assumed constant through the boundary layer at its value at the edge condition in equilibrium, and the edge Mach number $M_{e}$ is taken as the frozen edge Mach number. Inviscid linear stability is analyzed with the method described by Mack..$^{22}$ The temporal inviscid growth rate of a disturbance wave that travels in the direction of the cylinder axis is examined with assuming perfect gas. The components in the chordwise direction of all gradients of the mean flow quantities are neglected in the instability analysis Because of the highly cooled wall, only the higher modes are found 
unstable for all shot conditions examined, as already noted. Because the preceding analysis is based on perfect gas, two-dimensional, inviscid, and linear stability theory, neither the frequency nor the growth rate of the most strongly amplified disturbance may be necessarily accurate. Indeed, the frequency for the most strongly amplified disturbanceestimated with Eq. (2) is approximately two to three times higher than the peak frequency of the inviscid linear instability disturbance obtained here. One major cause is that the perfect gas boundary-layercalculation results in a thicker boundary layer than the BLIMPK calculation, that is, real gas calculation, does by a factor of 1.5-2. Nevertheless, the inviscid linear stability results give the correct order of magnitude for both the maximum amplification rate and the peak frequency, which is sufficient for our purpose here.

\section{Results and Discussion}

\section{0-Degree Sweepback Model}

The summary data on the model of 60-deg sweep angle are presented in the form of plots of Poll's Reynolds number $\bar{R} e^{*}$ for every shot vs freestream total enthalpy in Fig. 8. Because the boundary layer does not grow along the cylinder axis, the data for a particular shot condition can only show whether the boundary layer is laminar or turbulent and do not give the transition Reynolds number directly. Thus, we can conclude that the transition Reynolds number lies somewhere between the open symbols and the solid symbols, which represent laminar and turbulent boundary layer, respectively. Symbols + indicate that the state is intermittent, that is, transitional. For the nitrogen tests, the transition Reynolds number lies between 600 and 700, which is approximately the same value as that observed in a supersonic quiet wind-tunnel experiment, ${ }^{17}$ and no significant dependence on total enthalpy can be seen through the range from 2 to $15 \mathrm{MJ} / \mathrm{kg}$. The air data show a slightly higher transition Reynolds number than the nitrogen data, and, again, there appears to be no dependence on the total enthalpy. On the other hand, for the carbon dioxide data, some features can be clearly observed. The transition Reynolds number increases rapidly with total enthalpy from $\bar{R} e^{*} \approx 600-700$ at $h_{0} \approx 1 \sim 2 \mathrm{MJ} / \mathrm{kg}$ to $\bar{R} e^{*} \approx 1000$ at $h_{0} \approx 7 \mathrm{MJ} / \mathrm{kg}$. It does not increase further for total enthalpy higher than approximately $7 \mathrm{MJ} / \mathrm{kg}$. The transition Reynolds number dependence on the total enthalpy is similar to that obtained in the cone experiments, although a clear saturation of transition Reynolds number for carbon dioxide tests could not be seen in the cone experiments.

The edge Mach number varies from 3.3 to 4.0 throughout these experiments, depending on the gas species and freestream total enthalpy (Fig. 9). According to the results of other researchers ${ }^{17,30}$ on attachment line transition, the transition Reynolds number increases with edge Mach number. However, the edge Mach number in this experiment decreases with increasing total enthalpy, whereas the transition Reynolds number is observed to increase with total enthalpy for the carbon dioxide case. This indicates that a dominant effect other than that of edge Mach number exists in this case.

One might also be curious to see whether the effect of the wall temperatureratio can be an alternativeexplanation. This effect, however, cannot explain the trends in transition Reynolds number for the carbon dioxide case with the 60-deg sweep model. Indeed, the wallto-stagnation temperature ratio shown in Fig. 10, in which the wall temperature is taken to be constant at $300 \mathrm{~K}$, indicates only a small variation with different test gas used. Furthermore, the temperature ratio decreases less with increasing total enthalpy for carbon dioxide than for air or nitrogen, whereas the observed trend in transition Reynolds number is opposite.

It might give a good insight into the high-enthalpy effect issue to estimate the sound absorptionrate at the temperature of the reference condition, which represents an average condition in the boundary layer as mentioned before. Therefore, the sound absorption rate per wavelength $\alpha_{\text {relax }} \lambda$ at the reference-enthalpycondition is calculated at the frequency estimated as most strongly amplified [Eq. (2)], provided that the second mode is dominant (Fig. 11). In the case of carbon dioxide, this clearly shows the similarity between the trend of absorption rate and that of the transition Reynolds number with total enthalpy. The sound absorption rate increases with total enthalpy up

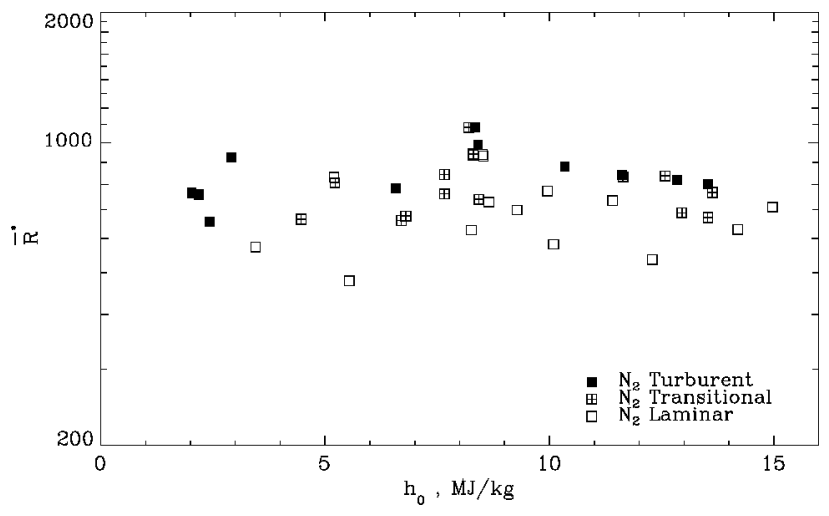

a) Nitrogen

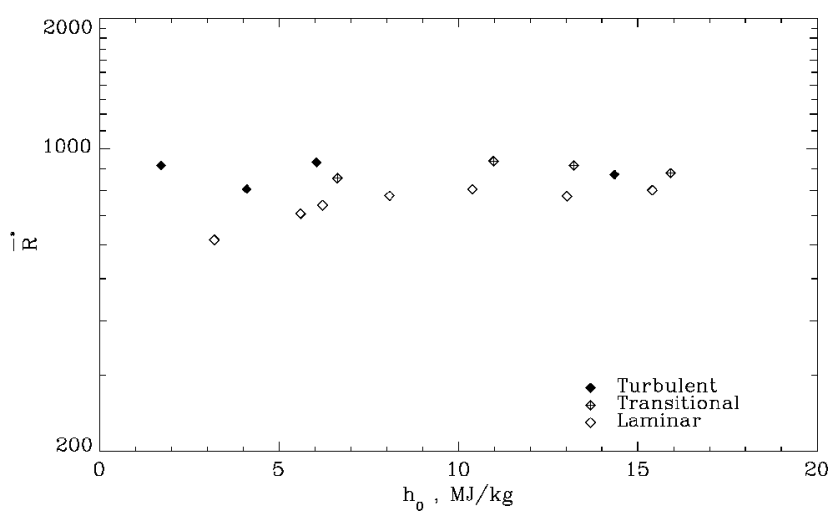

b) Air

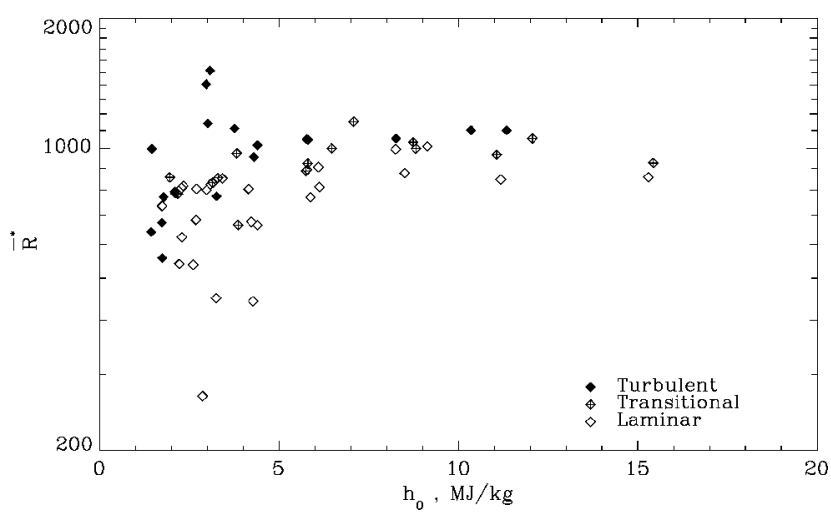

c) Carbon dioxide

Fig. 8 Transition Reynolds number $\overline{\boldsymbol{R}} e_{t}^{*}$ vs total enthalpy for nitrogen, air, and carbon dioxide observed on the $\Lambda=60 \mathrm{deg}$ model; no significant dependence is evident for nitrogen and air, whereas a strong effect is evident for carbon dioxide in the enthalpy range $h_{0}=1-7 \mathrm{MJ} / \mathrm{kg}$.

to about $7 \mathrm{MJ} / \mathrm{kg}$, and after that, it becomes nearly constant or even decreases slightly. This becomes clear from Fig. 1, which shows that the vibrational relaxation effect at the frequency of interest, $1-10 \mathrm{MHz}$, becomes dominant as the temperature reaches $2000 \mathrm{~K}$, which corresponds roughly to total enthalpy of 5-6 MJ/kg in this test situation. However, higher temperature causes carbon dioxide to begin to dissociate and the relaxation effects to remain roughly constant or even to decrease.

For the nitrogen case, the absorption rate due to relaxation is quite small compared with that in carbon dioxide, which is also consisten with the measured trend in transition Reynolds number. It comes from the fact that the vibrational excitation and dissociation for nitrogen become important at higher temperatures than for carbon dioxide. For the air experiments, the expected absorption rate is higher than in the nitrogen case. However, the magnitude is not significant. This is because the oxygen molecule is more easily vibrationally excited and the absorption peak frequency is closer to 


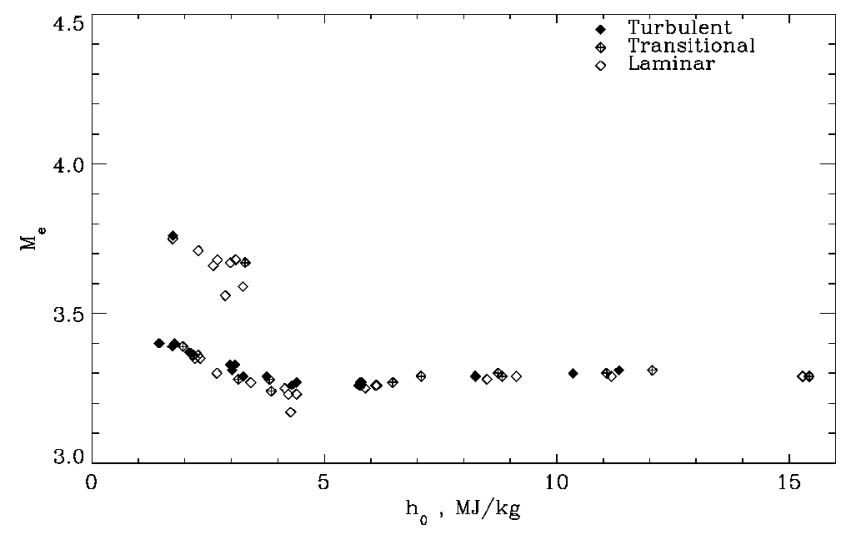

a) $\mathrm{CO}_{2}, \Lambda=60 \mathrm{deg}$

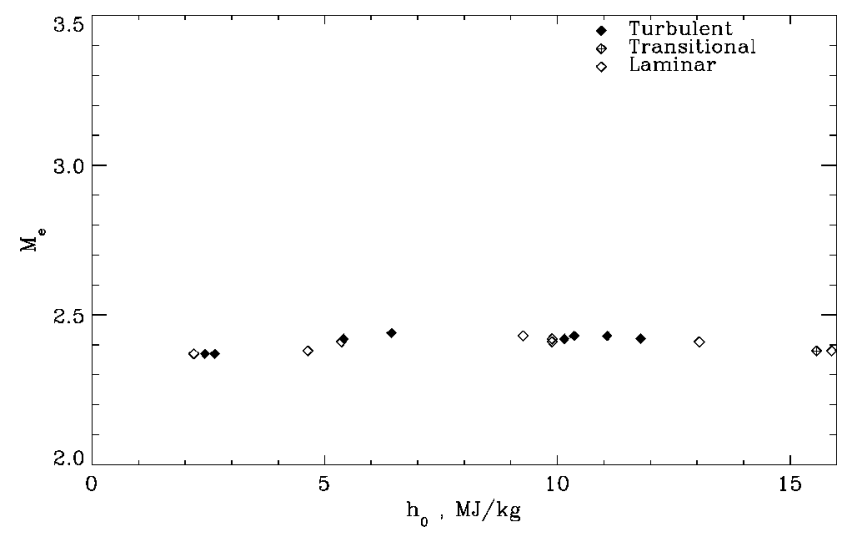

b) $\mathrm{CO}_{2}, \Lambda=45 \mathrm{deg}$

Fig. 9 Frozen edge Mach number vs total enthalpy for carbon dioxide tests; edge condition assumed both chemically and vibrationally in equilibrium.

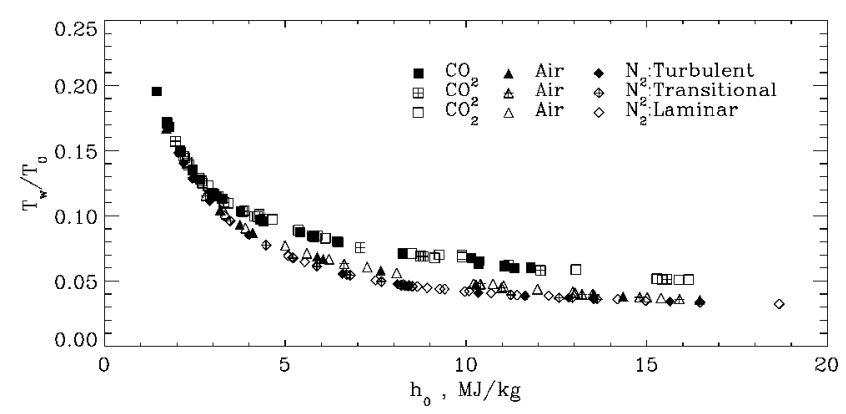

Fig. 10 Ratio of wall temperature (assumed at constant of $300 \mathrm{k}$ ) and stagnation temperature.

the most strongly amplified frequency, although the mole fraction of oxygen is relatively small and it has only one vibrational mode compared with four in carbon dioxide.

Although the trends of absorption rate and transition Reynolds number with total enthalpy are similar, one might have a question about the relative magnitude of absorption rate to the amplification rate of linear instability as a function of frequency. To compare these approximately, an inviscid linear stability analysis was carried out assuming perfect gas flow for both the mean flow profile and the disturbances. Amplification rates per cycle for several representative cases among nitrogen, air, and carbon dioxide shots are shown in Fig. 12 together with absorption rate per wave length. For carbon dioxide, the absorption rate due to vibrational relaxation of $\mathrm{CO}_{2}$ is quite strong compared with the amplification rate, for the midenthalpy conditions ( $\operatorname{shot} 2046 ; h_{0}=5.6 \mathrm{MJ} / \mathrm{kg}$ ). In contrast, for a lower enthalpy condition (shot $2035 ; h_{0}=1.63 \mathrm{MJ} / \mathrm{kg}$ ) because the two characteristic frequencies are completely different, that is, low Damkhöler number [Eq. (3)], absorption may not be

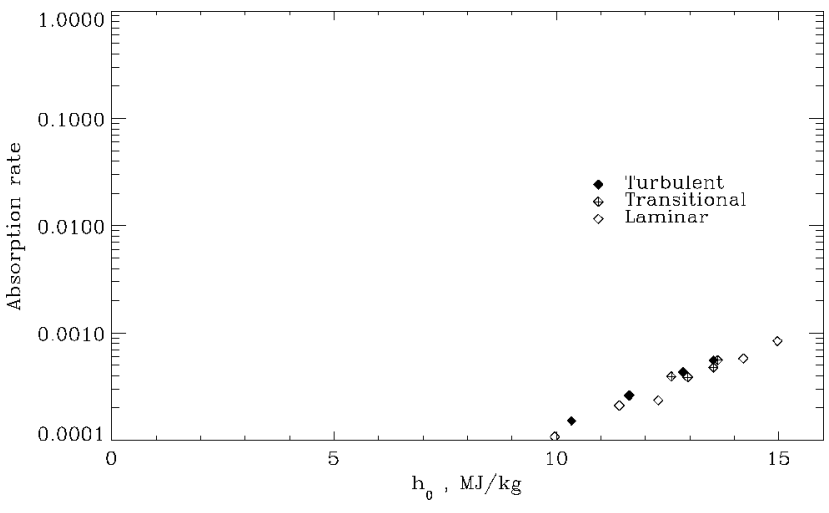

a) Nitrogen

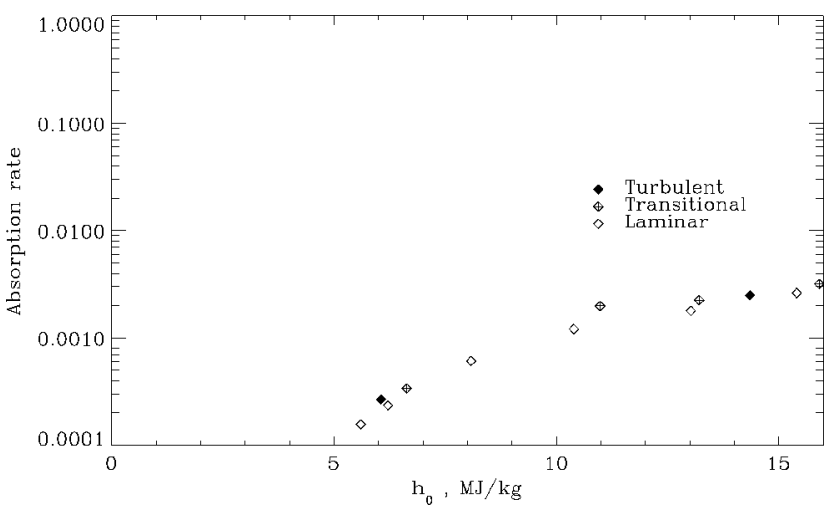

b) Air

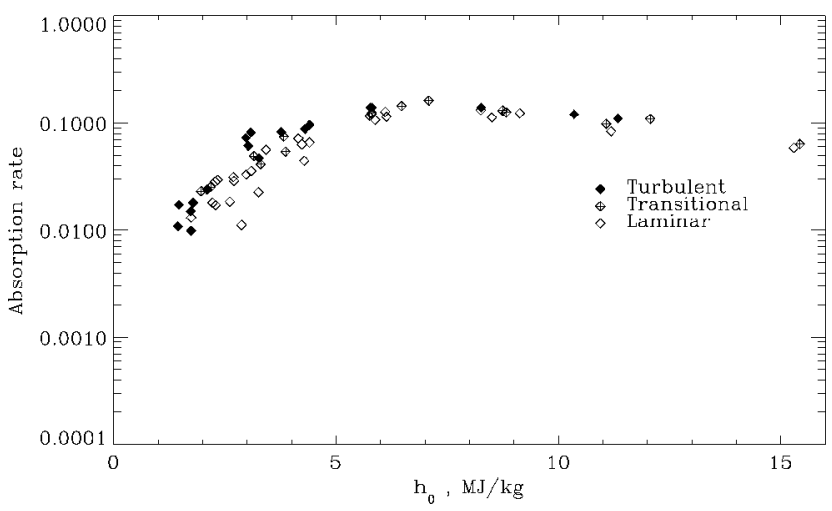

c) Carbon dioxide

Fig. 11 Sound attenuation rate per wave length evaluated at reference enthalpy conditions vs total enthalpy for $\Lambda=60$ deg model test conditions.

expected to affect transition significantly. At an even higher total enthalpy condition ( $\operatorname{shot} 2216 ; h_{0}=15.63 \mathrm{MJ} / \mathrm{kg}$ ), the absorption peak passes the second-mode frequency, and the peak absorption value is getting weaker due to $\mathrm{CO}_{2}$ dissociation. Remember that the instability analysis conducted here gives only approximate values for peak frequency due to the perfect gas assumption in the mean flow calculation. Nevertheless, we can see in Fig. 12 that the sound absorption rate at the estimated second-mode frequency is not negligible compared with the second-mode amplification rate. The large transition delay observed in the carbon dioxide tests can be consistently explained by the effect of relaxation of vibrational excitation, supposing the second-mode instability to be responsible.

On the other hand, nitrogen sound absorption due to vibrational relaxation has its peak so far from the most strongly amplified frequency estimated by inviscid linear stability that we might not expect any effect of relaxation on linear disturbance growth according to Fig. 12. Air cases are basically similar to nitrogen cases, except that oxygen vibration causes a slightly stronger absorption effect. 


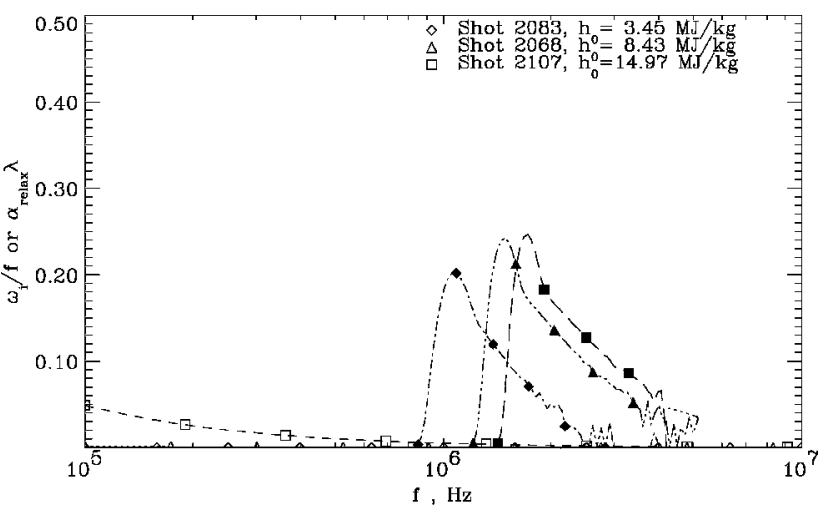

a) Nitrogen

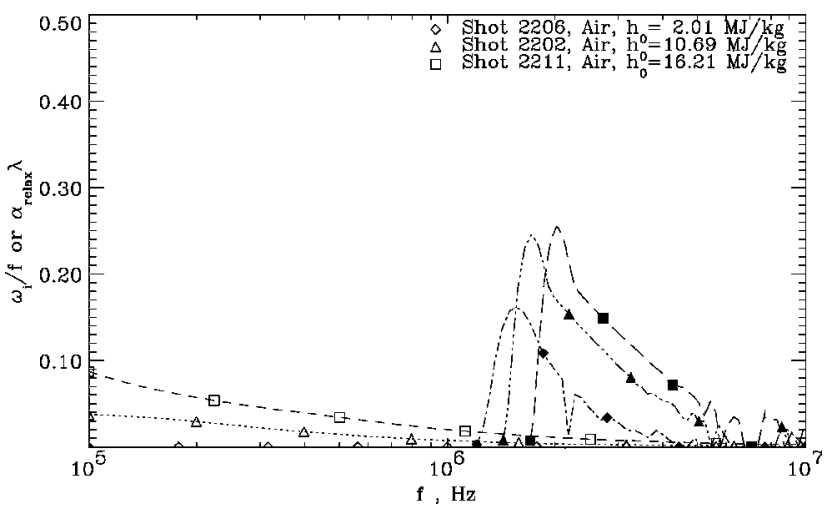

b) Air

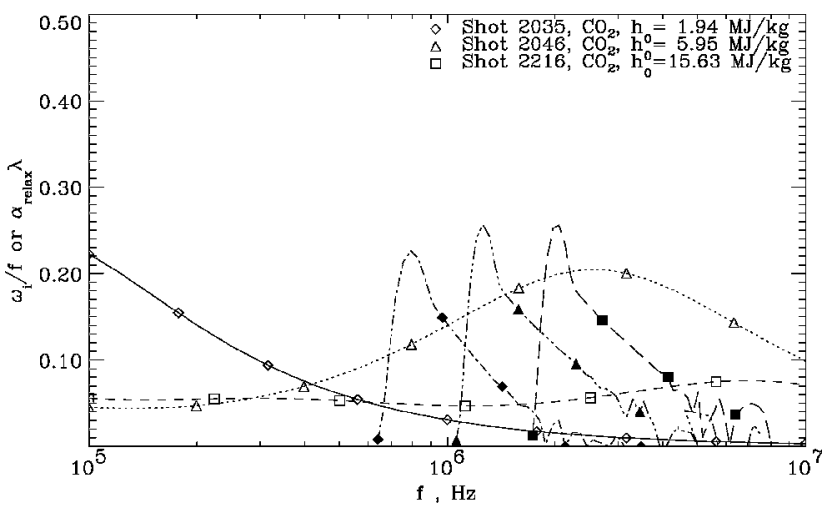

c) Carbon dioxide

Fig. 12 Comparison of the absorption rate per wavelength due to relaxation: $\diamond, \triangle, \square$, with the amplification rate per cycle from perfect-gas linear stability calculations; and $\bullet, \Delta, \square$, for several particular $T 5$ shots at 60-deg swept angle.

\section{5-Degree Sweepback Model}

Poll's Reynolds number $\bar{R} e^{*}$ for the test results with 45 -deg sweep angle are shown vs freestream total enthalpy in Fig. 13. For all gases, no significant feature in transition Reynolds number is recognized except an increase around a total enthalpy of $15 \mathrm{MJ} / \mathrm{kg}$ in carbon dioxide. The rest of the transition Reynolds numbers lie between 700 and 800 . At first sight, this result is somewhat unexpected because the absorption rate at the second-mode frequency, as shown in Fig. 14, is estimated to be even higher than for 60-deg sweep angle for all of the tests. Furthermore, the amplification rate per cycle is calculated to be even less than in the other case (Fig. 15). This unexpected result has not been explained by the authors. However, one possiblity is that, due to the relatively low edge Mach number $M_{e} \approx 2.1-2.4$ (Fig. 9), the finite Reynolds number might make the first mode unstable, in spite of the very highly cooled wall. Because the relaxation process is not expected to affect vorticity wave propagation, as we might see by an analogy with the bulk viscosity effect,

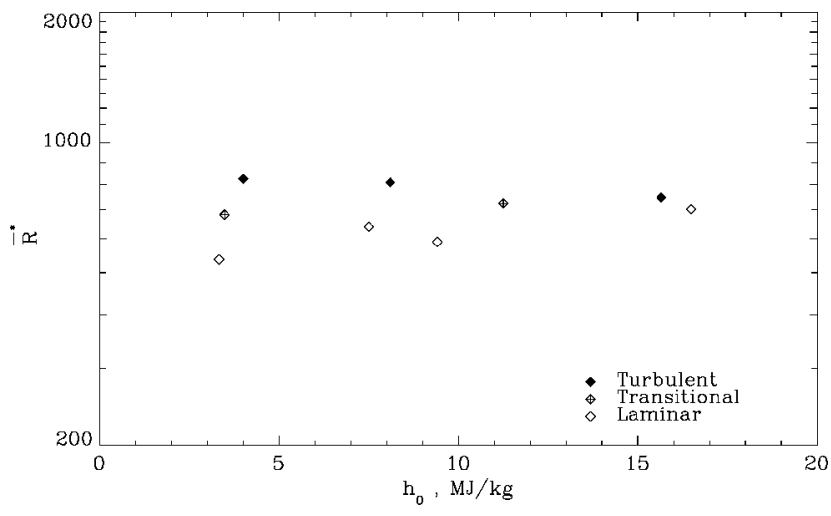

a) Nitrogen

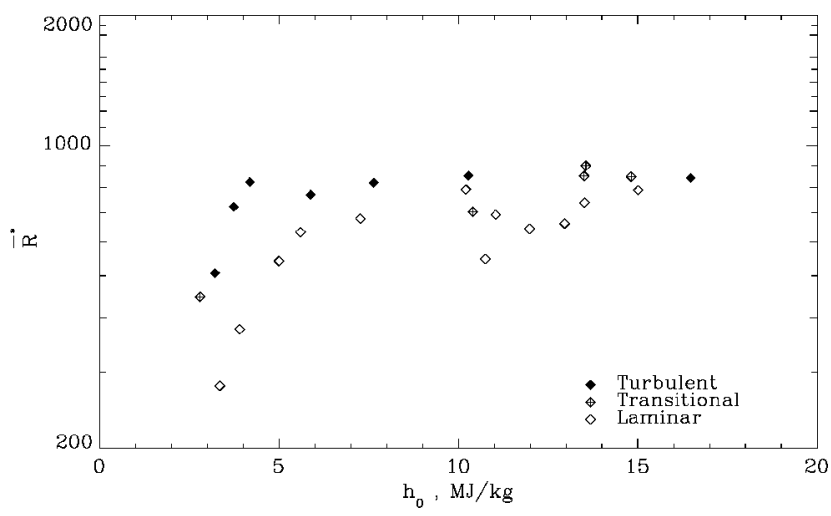

b) Air

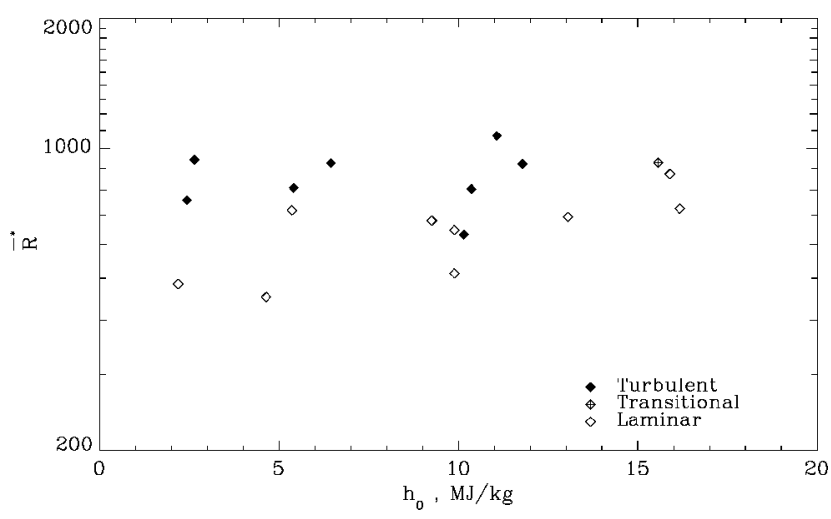

c) Carbon dioxide

Fig. 13 Transition Reynolds number $\overline{\boldsymbol{R}} e_{t}^{*}$ vs total enthalpy for nitrogen, air, and carbon dioxide observed on the $\Lambda=45 \mathrm{deg}$ model.

this could explain the absence of significant total enthalpy dependence. Another possibility is that the lower edge Mach number is expected to restrict the relative supersonic region where the second mode is trapped only in the vicinity of the wall, whereas the location where sound absorption rate was calculated remains approximately the same (relatively close to the boundary-layer edge). If so, the temperature at which absorption should be evaluated might be much lower than that of the reference condition, and it would weaken the relaxation effect a lot. These possibilities remain to be examined in the future.

It is important to realize that the mechanism of transition delay by relaxation claimed in this paper suggests the possibility of socalled high-enthalpy effects on transition in hypersonic flow that are quite different from those observed here. As already mentioned [Eq. (2)], the most strongly amplified frequency without chemistry can be basically determined only by edge velocity and boundarylayer thickness, whereas sound absorption effects depend additionally on representative temperature (for magnitude and frequency) and pressure (for frequency). These parameters might have quite 


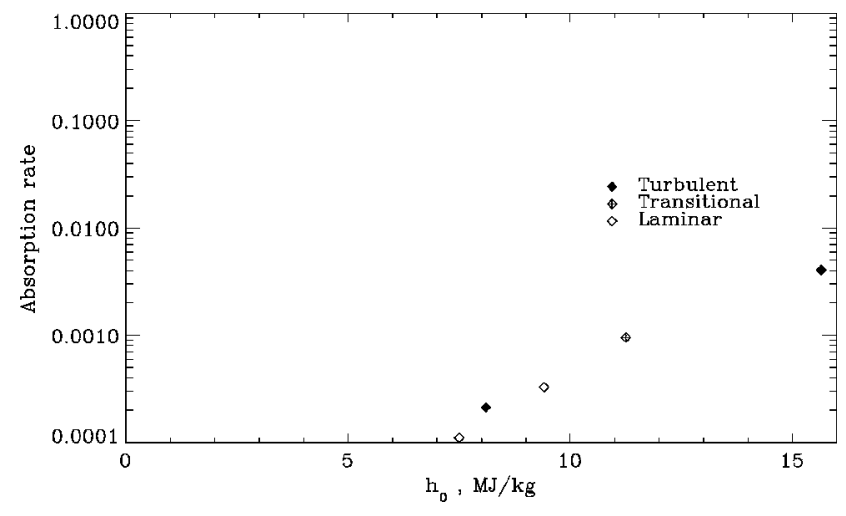

a) Nitrogen

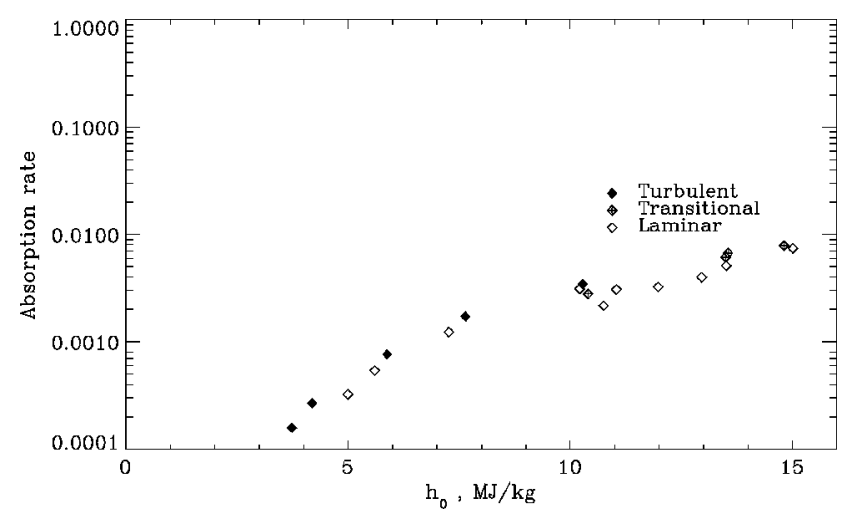

b) Air

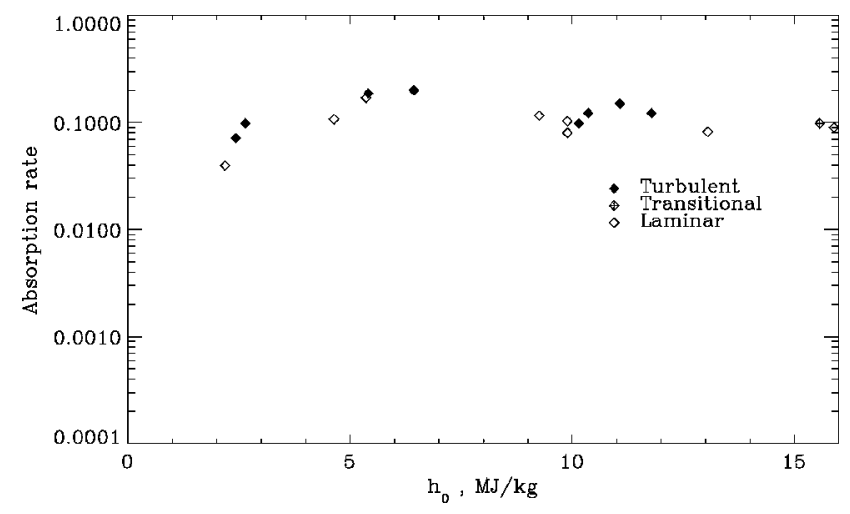

c) Carbon dioxide

Fig. 14 Sound attenuation rate per wavelength evaluated at reference enthalpy conditions vs total enthalpy for $\Lambda=45 \mathrm{deg}$ model test conditions.

different values, depending on freestream conditions, angle of attack, wall temperature, and geometry.

\section{Limitations of the Experiments}

As in most experimental studies, there are several limitations to the overall experiment, which should be addressed. An issue related to the limitations is the lack of knowledge of the noise spectrum radiated by the nozzle wall boundary layer in the $\mathrm{T} 5$ because the tunnel noise level is known to have a strong effect even on the trend of transition Reynolds number, as discussed recently in the review by Schneider. ${ }^{31}$ We can only assume the noise level in the T5 to be of order $3 \%$ in pitot pressure fluctuation, as He and Morgan ${ }^{1}$ measured in T4, a shock tunnel similar to T5, at relatively low frequencies (hundreds of kilohertz), although a limited measurement of pressure fluctuation in the T5 (Ref. 32) does not show the large peak of unknown origin that is the dominant fluctuation in the T4 measurement. At this time, neither the noise level in the T5 freestream at the frequencies of the most strongly amplified

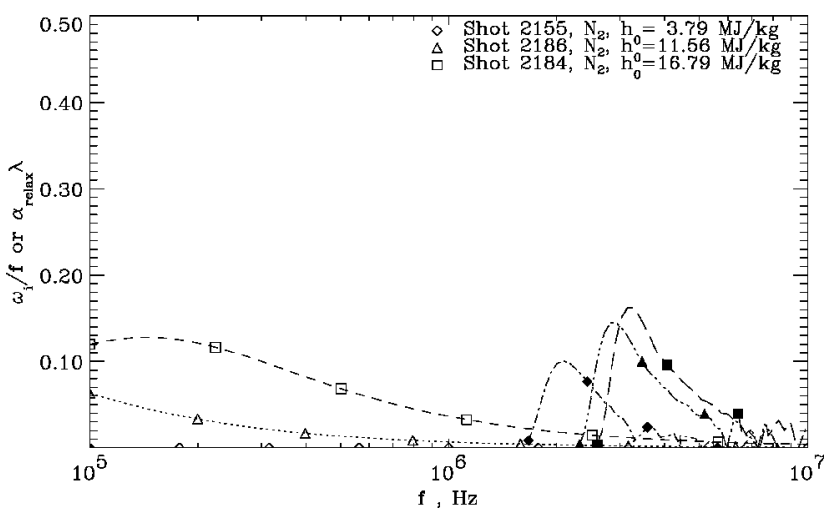

a) Nitrogen

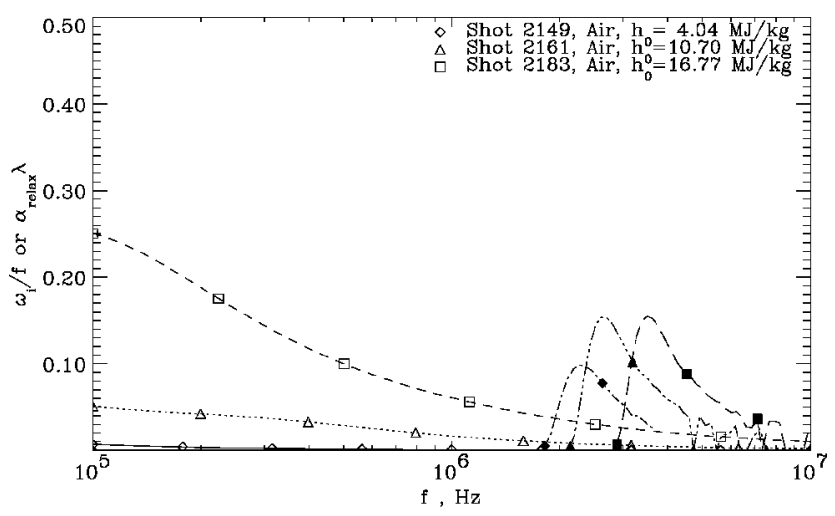

b) Air

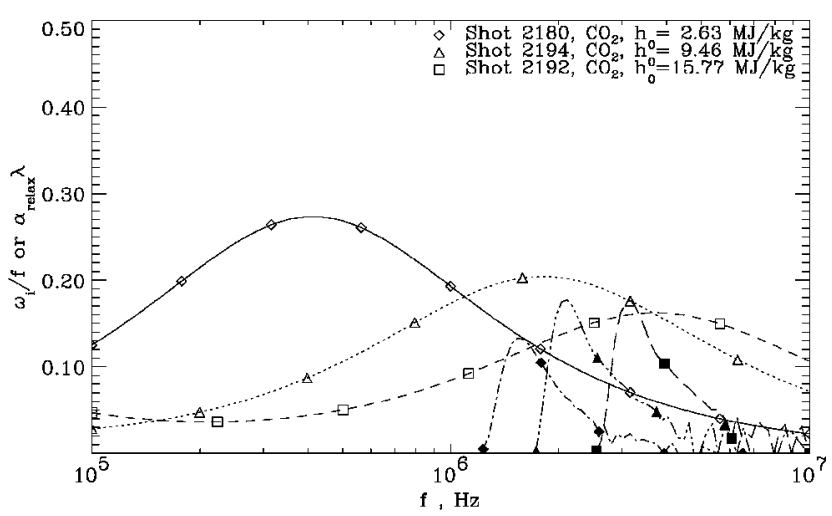

c) Carbon dioxide

Fig. 15 Comparison of the absorption rate per wavelength due to relaxation: $\diamond, \triangle, \square$, with the amplification rate per cycle from perfect-gas linear stability calculations; and $\bullet, \Delta, \varpi$, for several particular $T 5$ shots at 45-deg sweep angle.

disturbances nor the dependence of the noise spectrum on the total enthalpy are known.

\section{Conclusions}

A series of experiments on attachment line transition was conducted to study high-enthalpyeffects in the T5 hypervelocity shock tunnel, where the freestream noise level is unknown. The trend of transition Reynolds number with enthalpy, which is found to be similar to results from cone flow, shows a strong effect of transition delay for carbon dioxide tests on 60-deg sweptback cylinder model, although no significant effect can be seen for nitrogen and air. In all cases where relaxation effects are inactive, the transition Reynolds number is comparable with that observed in the quiet tunnel at NASA Langley, Research Center, and the relaxation effects increase it by as much as $50 \%$.

Sound absorption due to relaxation is calculated at the frequency estimated as most strongly amplified and at the reference enthalpy 
condition of the attachment-line boundary layer of the experiment. Comparison indicates a similar trend in enthalpy dependence between transition Reynolds number and absorption rate for carbon dioxide at 60 -deg sweep angle. This suggests that the dominant effect in delaying boundary-layertransition in the carbon dioxide case is vibrational relaxation, supposing that the responsible instability mode is the second mode. Linear inviscid stability analysis is also carried out by assuming perfect gas flow for both boundary-layer profile and disturbances. The comparisons between the magnitude of the strongest amplification rate and relaxation absorption rate show that they are of the same order of magnitude for carbon dioxide and that absorption is almost negligible for nitrogen and air, which supports the results of this experiment at 60-deg sweep angle according to the understanding for relaxation effects on transition examined here.

The results at 45-deg sweep angle indicate no significant highenthalpy effect on transition Reynolds number for all gases tested, in spite of the even larger sound absorption expected. The reason is still not clear, but one possibility is that relaxation does not affect vorticity disturbances that might be active at the relatively low edge Mach number at 45-deg sweep angle.

\section{References}

${ }^{1}$ He, Y., and Morgan, R. G., "Transition of Compressible High Enthalpy Boundary Layer Flow over a Flat Plate," Aeronautical Journal, Vol. 98, No. 971, 1994, pp. 25-34.

${ }^{2}$ Germain, P., and Hornung, H. G., "Transition on a Slender Cone in Hypervelocity Flow," Experiments in Fluids, Vol. 22, 1997, pp. 183-190.

${ }^{3}$ Adam, P., and Hornung, H. G., "Enthalpy Effects on Hypervelocity Boundary-LayerTransition: Ground Test and FlightData," Journal of Spacecraft and Rockets, Vol. 34, No. 5, 1997, pp. 614-619.

${ }^{4}$ Malik, M. R., and Anderson, E. C., "Real Gas Effects on Hypersonic Boundary-Layer Stability," Physics of Fluids A, Vol. 3, No. 5, 1991,pp. 803821.

${ }^{5}$ Stuckert, G., and Reed, H., "Linear Disturbances in Hypersonic, Chemically Reacting Shock Layers," AIAA Journal, Vol. 32, No. 7, 1994, pp. 13841393.

${ }^{6}$ Johnson, H. B., Seipp, T., and Candler, G. V., "Numerical Study of Hypersonic Reacting Boundary Layer Transition on Cones," Physics of Fluids, Vol. 10, No. 10, 1998, pp. 2676-2685.

${ }^{7}$ Clarke, J. F., and McChesney, M., The Dynamics of Real Gases, Butterworths, London, 1964, Chap. 6.

${ }^{8}$ Rasheed, A., "Passive Hypervelocity Boundary Layer Control Using an Ultrasonically Absorptive Surface," Ph.D. Dissertation, Graduate Aeronautical Labs., California Inst. of Technology, Pasadena, CA, Jan. 2001.

${ }^{9}$ Lighthill, M. J., "Viscosity Effects in Sound Waves of Finite Amplitude," Surveys in Mechanics, edited by G. K. Batchelor and R. M. Davies, Cambridge Univ. Press, Cambridge, England, U.K., 1956, pp. 250351 .

${ }^{10}$ Vincenti, W. G., and Kruger, C. H., Introduction to Physical Gas Dynamics, Krieger, Malabar, FL, 1965, Chap. 8.

${ }^{11}$ Fujii, K., and Hornung, H. G., "A Procedure to Estimate the Absorption Rate of Sound Propagating Through High Temperature Gas," California Inst. of Technology, GALCIT TR FM 01-4, Pasadena, CA, Aug. 2001.

${ }^{12}$ Stetson, K. F., "Hypersonic Boundary-Layer Transition," Advances in Hypersonics: Volume I, Defining the Environment, edited by J. J. Bertin, J. Periaux, and J. Ballmann, Birkhäuser, Boston, 1992, pp. 324-417.

${ }^{13}$ Eckert, E. R. G., "Engineering Relations for Friction and Heat Transfer to Surfaces in High Velocity Flow," Journal of the Aeronautical Sciences, Vol. 22, Aug. 1955, pp. 585-587.

${ }^{14}$ Poll, D. I. A., "Transition Description and Prediction in Three Dimensional Flows, Special Course on Stability and Transition of Laminar Flow, AGARD Rept. 709, March 1984, Chap. 5

${ }^{15}$ Poll, D. I. A., "The Development of Intermittent Turbulence on a Swept Attachment Line Including the Effects of Compressibility," Aeronautical Quarterly, Vol. 34, No. 1, 1983, pp. 1-23.

${ }^{16}$ Benard, E., Gaillard, L., and Alziary de Roquefort, T., "Influence of Roughness on Attachment Line Boundary Layer Transition in Hypersonic Flow," Experiments in Fluids, Vol. 22, No. 4, 1997, pp. 286-291.

${ }^{17}$ Creel, T. R., Beckwith, I. E., and Chen, F. J., "Transition on Swept Leading Edges at Mach 3.5," Journal of Aircraft, Vol. 24, No. 10, 1987 , pp. 710-717.

${ }^{18}$ Beckwith, I. E., and Gallagher, J. J., "Local Heat Transfer and Recovery Temperatires on a Yawed Cylinder at a Mach Number of 4.15 and High Reynolds Numbers," NASA TR R-104, 1961.

${ }^{19}$ Reynolds, W. C., "The Element Potential Method of Chemical Equilibrium Analysis: Implementation in the Interactive Program STANJAN," Technical Rept., Dept. of Mechanical Engineering, Stanford Univ., Stanford, CA, 1986.

${ }^{20}$ Wilke, C. R., "A Viscosity Equation for Gas Mixtures," Journal of Chemical Physics, Vol. 18, No. 4, 1950,pp. 517-519.

${ }^{21}$ Poling, B. E., Prausnitz, J. M., and O'Connell, J. P., The Properties of Gases and Liquids, 5th ed., McGraw-Hill, New York, 2001, Chap. 9.

${ }^{22}$ Mack, L. M., "Boundary-Layer Stability Theory," Special Course on Stability and Transition of Laminar Flow, AGARD Rept. 709, March 1984, Chap. 3.

${ }^{23}$ Van Driest, E. R., "Calculations of the Stability of the Laminar Boundary Layer in a Compressible Fluid on a Flat Plate with Heat Transfer," Journal of the Aeronautical Sciences, Vol. 19, No. 13, 1952, pp. 801-812.

${ }^{24}$ Dorrance, W. H., Viscous Hypersonic Flow, McGraw-Hill, New York, 1962, Chap. 5

${ }^{25}$ Tong, H., Buckingham, A. C., and Morse, H. L., "Nonequilibrium Chemistry Boundary Layer Integral Matrix Procedure,” NASA CR-134039, July 1973

${ }^{26}$ Cooke, J. C., "An Axially Symmetric Analogue for General ThreeDimensional Boundary Layers," British Aeronautical Research Council, TR R\&M 3200, London, June 1959.

${ }^{27}$ Chen, Y.-K., Henline, W. D., Stewart, D. A., and Candler, G. V., "Navier-Stokes Solutions with Surface Catalysis for Martian Atmospheric Entry," Journal of Spacecraft and Rockets, Vol. 30, No. 1, 1993, pp. 32-42.

${ }^{28}$ Bertolotti, F.P., "The Influence of Rotational and Vibrational Energy Relaxation on Boundary-Layer Stability," Journal of Fluid Mechanics, Vol.372, 1998, pp. 93-118.

${ }^{29}$ Reshotko, E., and Beckwith, I. E., "Compressible Laminar Boundary Layer over a Yawed Infinite Cylinder with Heat Transfer and Arbitrary Prandtl Number,' NACA Rept. 1379, 1958

${ }^{30}$ Murakami, A., Stanewsky, E., and Krogmann, P., "Boundary-Layer Transition on Swept Cylinders at Hypersonic Speeds," AIAA Journal, Vol. 34, No. 4, 1996, pp. 649-654.

${ }^{31}$ Schneider, S. P., "Effect of High-Speed Tunnel Noise on LaminarTurbulent Transition," Journal of Spacecraft and Rockets, Vol. 38, No. 3, 2001, pp. 323-333.

${ }^{32}$ Davis, J. P., "High-Enthalpy Shock/Boundary-Layer Interaction on a Double Wedge," Ph.D. Dissertation, Graduate Aeronautical Labs., California Inst. of Technology, Pasadena, CA, 1999. 